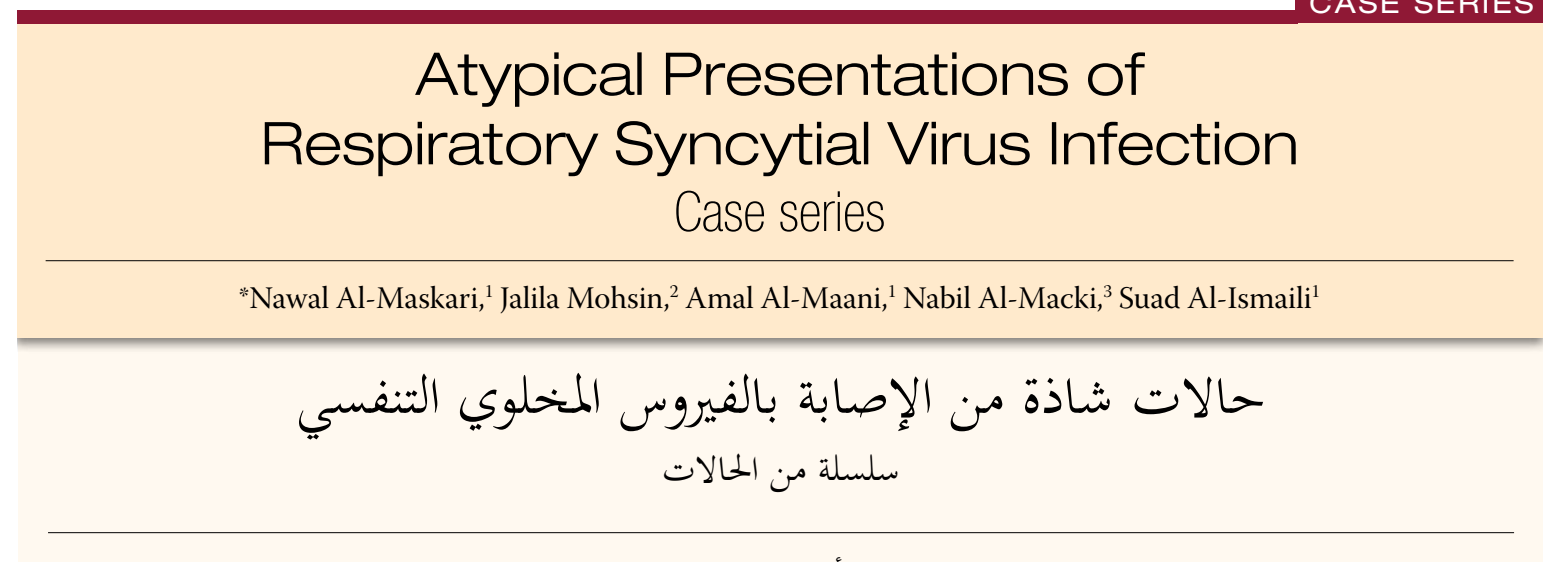

نوال المسكرية، جليلة محسن، أمل المعانية، نبيل مكي، سعاد الإسماعيلية

\begin{abstract}
The respiratory syncytial virus (RSV) usually causes a lower respiratory tract infection in affected patients. RSV has also been infrequently linked to extrapulmonary diseases in children. We report four children who had unusually severe clinical manifestations of RSV infections requiring critical care admission. These patients presented to the Royal Hospital, Muscat, Oman, in December 2013 with acute necrotising encephalopathy (ANE), acute fulminant hepatic failure with encephalopathy, pneumatoceles and croup. A unique presentation of ANE has not previously been reported in association with an RSV infection. All patients had a positive outcome and recovered fully with supportive management.
\end{abstract}

Keywords: Respiratory Syncytial Virus Infections; Epidemiology; Encephalitis; Hepatitis; Croup; Case Series; Oman.

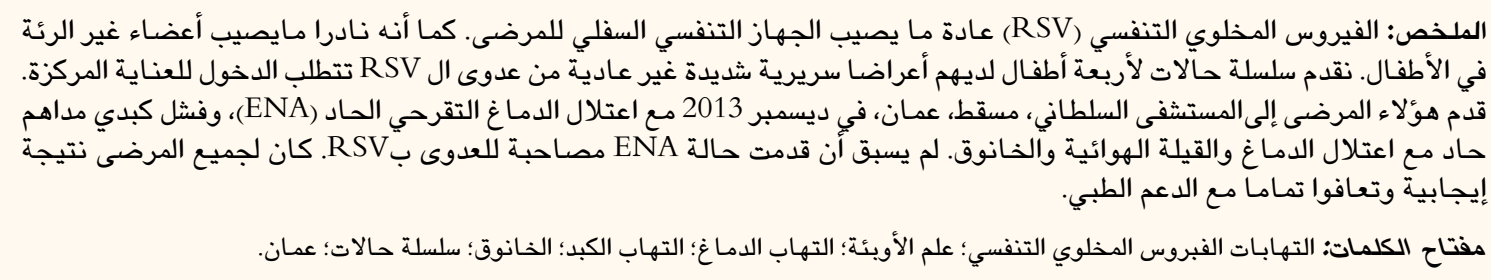

$\mathrm{T}$ HE RESPIRATORY SYNCYTIAL Virus (RSV) belongs to the Paramyxoviridae family and, along with the recently identified human metapneumovirus, belongs to the Pneumovirinae subfamily. RSV is further classified into subtypes A and B; subtype A usually causes a more severe form of the disease. ${ }^{1}$ Both subtypes occur more commonly in cold weather and rainy seasons. ${ }^{2}$ Around the world, it has been noted that RSV is highly prevalent in fiveyear-old children. ${ }^{3}$ In the United States, 4-5 million children younger than four years of age annually contract RSV infections and 125,000 require hospital admission. ${ }^{4}$ In most cases, the virus is not fatal; however, in certain groups of high-risk patients, an RSV infection can lead to a more severe morbidity or result in death. ${ }^{5}$ In order to manage affected patients appropriately, clinicians need to be aware of severe intrapulmonary and extrapulmonary manifestations of RSV infections. ${ }^{6}$

RSV mainly causes lower respiratory tract infections in children younger than two years of age or upper respiratory tract infections in older children and adults. ${ }^{5,6}$ RSV rarely causes severe extrapulmonary manifestations like cerebellitis, encephalitis, fatal interstitial myocarditis, hepatitis or Reye's syndrome. ${ }^{6-9}$ This case series reports four children admitted to the critical care area of the Royal Hospital, Muscat, Oman, in December 2013 with severe and rare manifestations of RSV. All patients had a positive outcome and made a full recovery.

\section{Case One}

A previously healthy 11-month-old female infant was admitted to the Royal Hospital in December 2013 with a three-day history of fever, a runny nose and cough followed by drowsiness and respiratory distress. She had been in contact with her sibling who had an upper respiratory tract infection. A physical examination revealed fever, drowsiness and response only to painful stimuli. She was tachypnoeic, had marked hepatomegaly $(8 \mathrm{~cm}$ below the costal margin with a liver span of $13 \mathrm{~cm}$ ) and her spleen was not palpable.

Investigations showed a normal complete blood count $(\mathrm{CBC})$, high aspartate transaminase (AST) 

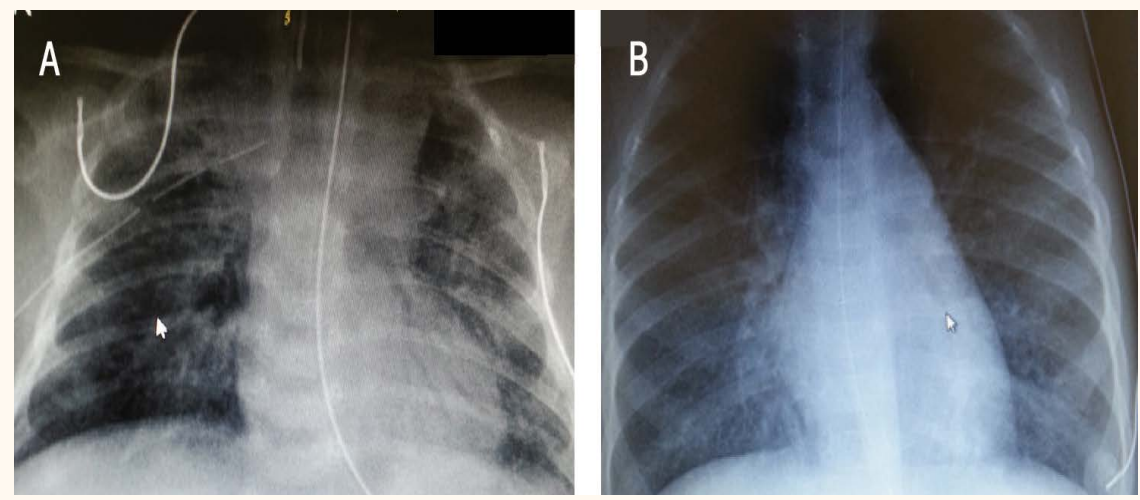

Figure 1 A\&B: Chest X-rays of an 11-month-old female infant (case one) with respiratory syncytial virus infection showing (A) right upper lobe consolidation and (B) complete consolidation resolution five days after admission.

levels of 9,000 IU/L (normal range: 5-60 IU/L), normal alkaline phosphatase levels of $163 \mathrm{IU} / \mathrm{L}$ (normal range: 90-210 IU/L), high alanine transaminase levels of $52 \mathrm{IU} / \mathrm{L}$ (normal range: $0-40 \mathrm{IU} / \mathrm{L}$ ) and normal albumin levels of $37 \mathrm{~g} / \mathrm{L}$ (normal range: $35-50 \mathrm{~g} / \mathrm{L}$ ). The patient had a deranged coagulation profile, a prothrombin time of 27.9 seconds (normal range: 9.1-11.6 seconds), activated partial thromboplastin time of 40.4 seconds (normal range: 27.3-39.1 seconds), fibrinogen 1 levels of $0.03 \mathrm{~g} / \mathrm{L}$ (normal range: 1.5-4.2 g/L) and a thrombin time of 23.80 seconds (normal range: 12-16 seconds). She was diagnosed with acute fulminant hepatic failure with encephalopathy.

A nasopharyngeal swab viral multiplex polymerase chain reaction (PCR) test revealed a positive result for RSV and a negative result for other respiratory viruses, including influenza $\mathrm{A}$ and $\mathrm{B}$, coronaviruses, parainfluenza viruses, rhinoviruses, adenoviruses, enteroviruses, paraechoviruses, human metapneumovirus, bocavirus and Mycoplasma pneumoniae. Plasma PCR tests were negative for herpes simplex virus and human immunodeficiency virus. Hepatitis E immunoglobulin (Ig) M, hepatitis A $\operatorname{IgG}$ and $\operatorname{IgM}$, hepatitis B surface antigen and hepatitis
$\mathrm{C}$ virus antibodies were negative. No growth was observed on blood and urine cultures. The metabolic work-up showed normal ammonia and urine organic acid with mildly elevated levels of methionine and phenylalanine; these were likely secondary to the hepatic dysfunction.

Five days after admission, a repeat $\mathrm{CBC}$ showed leukocytosis with lymphocytosis. A chest X-ray (CXR) revealed right upper lobe consolidation [Figure 1A]. Computed tomography (CT) of the head was normal. The patient was stabilised in the Paediatric Intensive Care Unit (PICU) and did not require ventilation. She was prescribed cefotaxime and acyclovir empirically. Five days later, her AST levels dropped to 1,500 IU/L. She improved gradually and regained a normal level of consciousness. Within seven days her liver had returned to a normal size and her CXR showed complete resolution of the consolidation [Figure 1B].

\section{Case Two}

A nine-year-old boy diagnosed with a recurrent medulloblastoma was admitted to the Royal Hospital in December 2013 with a two-day history of fever,
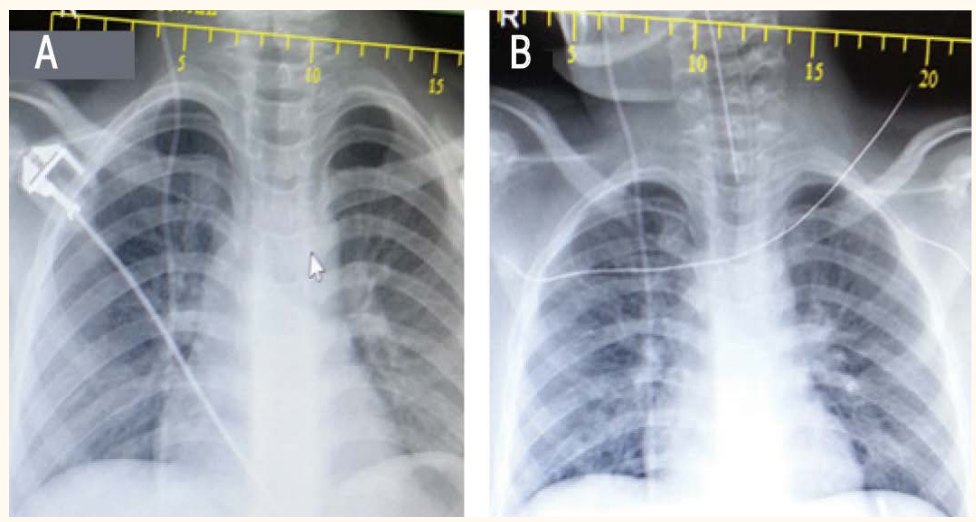

Figure 2 A\&B: Chest X-rays of a nine-year-old boy (case two) with respiratory syncytial virus infection showing (A) upper airway narrowing which required (B) intubation. 

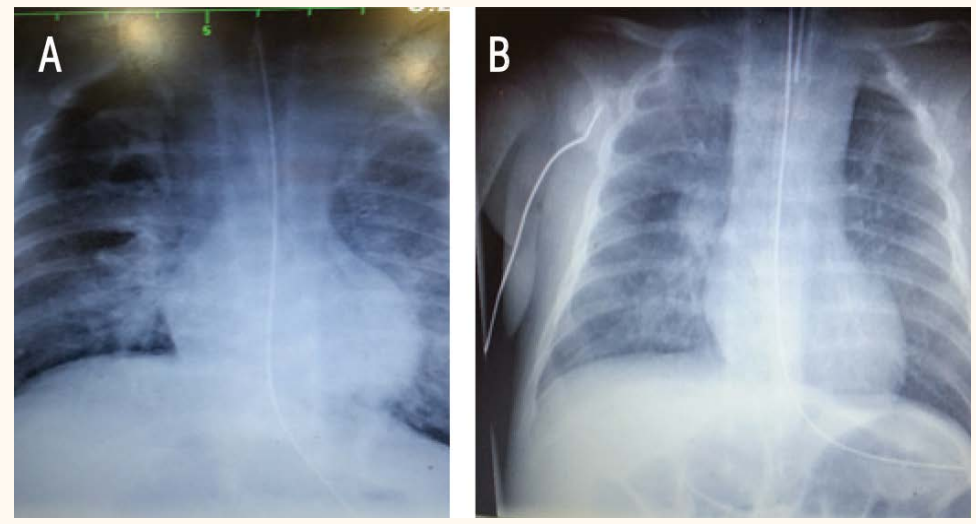

Figure 3 A\&B: Chest X-rays of a 33-day-old male infant (case three) with respiratory syncytial virus infection showing (A) right-side pneumatoceles on admission and (B) the resolution of the pneumatoceles four days after admission.

stridor, a barking cough and respiratory distress. On examination, he was febrile with bilateral equal breath sounds and an expiratory wheeze. He had received high-dose chemotherapy seven days prior to admission. His investigations showed high levels of C-reactive protein. A nasopharyngeal swab viral multiplex PCR test was positive for RSV and negative for other viruses. The patient's CBC showed severe leukopenia $0.1 \times 10^{9} / \mathrm{L}$ (normal range: $1.4-9.0 \times 10^{9} / \mathrm{L}$ ) with no neutrophils. His CXR showed a narrowing of the upper third of the trachea [Figure 2A] with compensatory hyperinflation and no consolidation. The patient was diagnosed with croup and was prescribed adrenaline and dexamethasone. He had severe stridor requiring intubation [Figure 2B] and a three-day course of ventilation. Six days later, a repeat CBC, neck X-ray and CXR showed resolution of the aforementioned signs. He completely recovered and was discharged seven days after admission.

\section{Case Three}

A 33-day-old male infant was admitted to the PICU at the Royal Hospital in December 2013 with a twoday history of fever, cough and poor feeding. On examination, he was febrile, tachycardic, tachypnoeic and required $3 \mathrm{~L}$ of oxygen via nasal prongs for maintained saturation. Investigations showed a normal CBC, urea electrolyte panel, bone profile and urine analysis. A CXR revealed bronchopneumonia with multiple pneumatoceles [Figure 3A]. The day after admission, he became distressed and was found to be hypotensive with right pneumothorax. He required intubation, ventilation, intercostal drainage (ICD) catheter insertion and inotropic support for three days. He began to improve on the fourth day of admission and the ICD catheter was removed and the inotropic support discontinued. He was extubated on the same day. The endotracheal secretion showed positive Haemophilus influenzae growth. A nasopharyngeal swab viral multiplex PCR test was positive for RSV and negative for other viruses. For a period of two weeks, he received intravenous ceftriaxone and clindamycin followed by oral clindamycin. A repeat CXR on the $12^{\text {th }}$ day of admission showed resolved pneumonia and a pneumatocele. On the $14^{\text {th }}$ day of admission, another CXR showed complete resolution of the pneumatoceles [Figure 3B]. On the same day, he was discharged on oral clindamycin to complete the fourweek course of treatment.

\section{Case Four}

A previously healthy six-year-old female was admitted to a peripheral hospital in Oman in December 2013 with a one-day history of fever, runny nose, cough and lethargy, followed by altered sensorium and an inability to walk. One day prior to admission, she received an oral antibiotic from a local health centre. On examination, she was drowsy, mute, febrile and unable to stand or walk without support. She did not have any meningeal signs, although a lumbar puncture showed significant leukocytosis (red blood cell count [RBC]: 10,000/UL; white blood cell count [WBC]: 50/UL; neutrophils: 70\%). Her cerebrospinal fluid (CSF) glucose level was $4.5 \mathrm{mmol} / \mathrm{L}$ and her protein level was $0.4 \mathrm{~g} / \mathrm{L}$. Her CSF Gram stain, bacterial antigen and culture stains were negative. A viral study was not performed. A nasopharyngeal swab viral multiplex PCR test was positive for RSV and negative for other viruses and Mycoplasma. The patient was suspected to have meningoencephalitis and was prescribed ceftriaxone, vancomycin and acyclovir empirically. On the same day, the patient developed recurrent abnormal movements-sudden twisting of the head to the right side, blankness in the eyes and generalised 

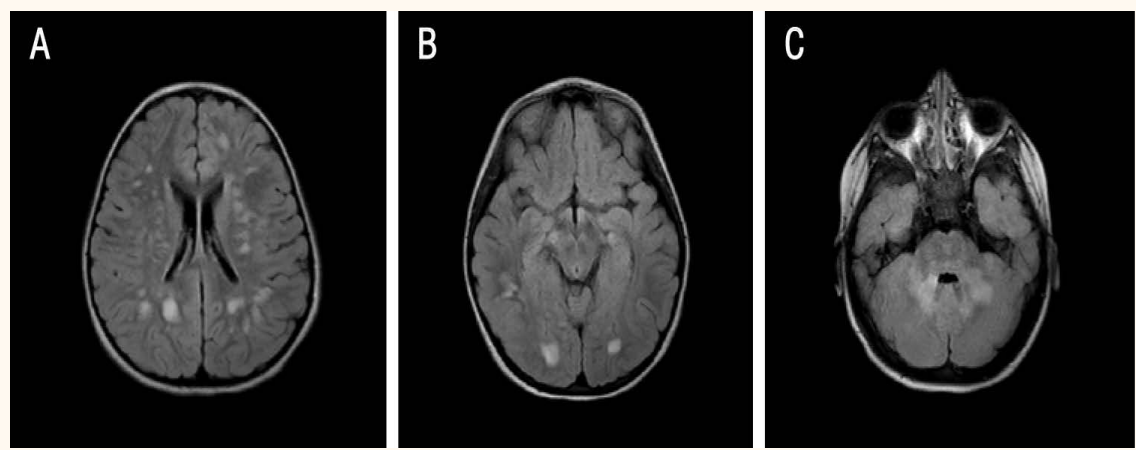

Figure 4 A\&B: Axial fluid-attenuated inversion recovery magnetic resonance images of the brain in a six-year-old female (case four) with respiratory syncytial virus infection showing (A) non-confluent white matter hyperintense foci involving the centrum semiovale, periventricular white matter and internal capsules, (B) hyperintense lesions involving the anterior midbrain and $(\mathbf{C})$ hyperintense lesions involving the cerebellar peduncles.

body stiffness which would last for a few seconds. These movements were eased by the administration of diazepam. When assessed, the patient scored between 8-11 on the Glasgow coma scale.

On the subsequent day, the patient was transferred to the PICU at the Royal Hospital for further evaluation and management. On arrival, she was obtunded, only opened her eyes to painful stimuli, did not respond to verbal commands and showed some withdrawal of the flexor response of the limbs to deep painful stimuli. There were bilateral pyramidal tract signs involving both the upper and lower limbs. She was ventilated due to her low Glasgow coma score and a depressed gag reflex. She developed a severe allergic skin reaction to ceftriaxone and was subsequently prescribed meropenem instead, although she continued to receive acyclovir. Upon her admission to the PICU, results of a brain CT scan were normal. Fluidattenuated inversion recovery magnetic resonance T2 imaging of the brain on the second day of admission revealed multiple subcortical white matter hyperintense lesions involving the periventricular white matter, internal capsule, left anterior midbrain, corpus callosum, posterior brainstem tract, cerebellar peduncles and left optic nerve [Figure 4]. This was suggestive of acute necrotising encephalopathy (ANE). She was treated with intravenous methylprednisolone pulse therapy for five days. As no improvement was seen from the methylprednisolone treatment, this was followed by intravenous immunoglobulin at a dosage of $2 \mathrm{~g} / \mathrm{kg}$ over the next five days.

The patient had multiple episodes of generalised tonic posturing and was treated with phenytoin and levetiracetam. As there were no clinical signs of improvement, a lumbar puncture was repeated on day nine of admission, which showed a negative culture with normal analysis (WBC: 5/UL; RBC: 20/UL; glucose: $4.6 \mathrm{mmol} / \mathrm{L}$; proteins: $0.2 \mathrm{~g} / \mathrm{L})$. The patient's
CSF viral PCR study was negative for herpes simplex viruses 1 and 2, enteroviruses, varicella zoster virus and mumps virus. Blood serology showed positive Mycoplasma IgG and negative IgM and IgA. On the $11^{\text {th }}$ day of admission, the patient received five sessions of plasmapheresis over the following seven days. She showed dramatic clinical improvement and was extubated at the end of the third week of admission.

With an extensive rehabilitation programme, the patient made excellent progress. When she was discharged, despite mild left upper limb weakness, she had normal speech, cognitive function and could walk with support. She was advised to continue physiotherapy. Three months after discharge, she was seen in a paediatric neurology outpatient clinic and was found to have no neurological deficits.

\section{Discussion}

Almost all children acquire a RSV infection by the second year of life. A minority (0.5-2.0\%) of infected children will require admission and most of them will be less than six months old. ${ }^{5}$ All of the patients in this case series were asymptomatic apart from upper respiratory tract infections for two days prior to admission. Three of the children were unique in that they had a complicated course with an extrapulmonary manifestation of RSV infection, while the remaining patient had such a severe lower respiratory tract infection that he was found to have a pneumatocele in association with RSV. Only one child was known to be immunocompromised; the other three had no known risk factors.

Pneumatoceles are caused primarily by Staphylococcus aureus, although they may also be associated with other pathogens like Streptococcus pneumoniae, H. influenzae and Klebsiella pneumoniae. ${ }^{10}$ Pneumatoceles are a very rare complication of RSV bronchiolitis. ${ }^{11}$ 
Almost 85\% of RSV pneumatoceles resolve completely and spontaneously. ${ }^{12}$ Lobar emphysema may also be associated with RSV pneumonia. ${ }^{12}$ One patient (case three) had symptoms of a viral infection with a runny nose and cough, then developed pneumonia and a pneumatocele which completely resolved within two weeks. Endotracheal secretions were positive for RSV PCR findings and $H$. influenzae non-type B culture, but his blood culture was negative. In this patient, it is likely that RSV was the primary infection; $H$. influenzae may have been a colonisation or secondary infection. Previous research has shown that serious bacterial infections are present in $0.6-1.2 \%$ of children admitted with RSV infections. ${ }^{13}$ To determine the aetiology of the pneumatoceles and pneumonia in the previously reported patient (case three), a lung biopsy was necessary. However, it was not carried out as the patient had improved; as such, a biopsy would not have added to the clinical management of the case.

Acute laryngeal croup is most often associated with parainfluenza viruses, RSV, rhinoviruses and enteroviruses. ${ }^{14}$ Usually croup presents in children of five years of age or younger. Unusually, one patient in this case series (case two) had severe croup due to RSV at the age of nine years; this can be explained by his secondary immunodeficiency following chemotherapy. Acute fulminant hepatic failure with encephalopathy is another rare atypical manifestation of RSV infection. ${ }^{15}$ Acute hepatitis can be a manifestation of other viruses such as the hepatitis, EpsteinBarr, cytomegalovirus and influenza viruses. One patient in this series (case one) had acute fulminant hepatic failure with encephalopathy and deranged coagulation. A liver biopsy was not performed; with supportive management, she recovered both clinically and biochemically within one week.

ANE is a rare, rapidly progressive, potentially fatal, parainfectious encephalopathy with specific neuroimaging findings that affect mainly healthy children between five months and 11 years of age. ANE seems to be more prevalent in children from Japan. ${ }^{16}$ It has a high mortality rate, reaching up to $30 \%$, with a severe neurological handicap occurring in $15 \%$ of survivors. ${ }^{17}$ ANE has been reported in patients with influenza types A and B, parainfluenza viruses, enteroviruses, reoviruses and $M$. pneumoniae. ${ }^{16-20}$ However, to the best of the authors' knowledge, ANE has not previously been reported in the English literature in association with RSV infections. The pathogenesis of RSV-related encephalitis is still not yet fully understood. However, it has been hypothesised that RSV may enter into the central nervous system through the haematogenous/blood-brain barrier route or through invasion with the release of several humoral neurotoxic cytokine mediators. ${ }^{21}$
It has been shown that treatments for ANE have limited efficacy. ${ }^{17}$ A recent report showed that treatment with steroids within the first 24 hours for three children without brainstem involvement improved outcomes, whereas the use of non-steroidal anti-inflammatory drugs has been associated with a poor prognosis and increased mortality rates. ${ }^{17}$ Methylprednisolone pulse therapy and high-dose $\gamma$-globulin treatment have been used to modulate immune-mediated neurovascular and cell injury. ${ }^{17}$ Plasma exchange can be used in patients who respond poorly to corticosteroids in order to modulate immune-mediated neurovascular and cell injuries. Although no data from randomised trials are available, successful treatment with plasma exchange has been reported. ${ }^{17,22,23}$ Alternative treatments for RSV infections that have been initiated in an attempt to improve mitochondrial function include carnitine, coenzyme $\mathrm{Q}_{10}$ and pyridoxine; hypothermia has also been tried as a treatment modality. ${ }^{17}$ The use of these interventions has been associated with a decrease in mortality rate (30\% to $15 \%) .{ }^{17}$ Currently, there is no clear evidence that the use of ribavirin improves the clinical outcome of critically ill infants with RSV infections. ${ }^{24}$

One patient in this case series (case four) had encephalopathy which rapidly progressed within 48 hours preceded by an upper respiratory tract infection. The respiratory viral panel showed positive RSV PCR results with negative findings for other viruses and M. pneumoniae. The patient was managed with methylprednisolone, plasmapheresis and extensive physiotherapy. She improved gradually and had fully recovered within three months. In this case, the most likely cause of ANE was the RSV infection. It is very difficult to confirm that ANE is caused only by RSV in the absence of a brain biopsy. A brain biopsy was not performed on this patient as it was thought to be an invasive procedure and the patient was in an unstable condition. Clinicians should consider ANE in cases of RSV infection with rapid progressive encephalopathy and start aggressive management accordingly.

\section{Conclusion}

In healthy children, an RSV infection usually causes a mild respiratory tract infection; however, some patients present with rare atypical and severe manifestations. Testing for RSV in these cases is therefore indicated. Clinicians should have a high suspicion of ANE when treating a patient with a respiratory infection and acute neurological manifestations. Early recognition of ANE is important for the initiation of effective supportive treatment in order to improve patient outcomes. 


\section{References}

1. Collins PL, Graham BS.Viral and host factors in human respiratory syncytial virus pathogenesis. J Virol 2008; 82:2040-55. doi: 10.1128/JVI.01625-07.

2. Omer SB, Sutanto A, Sarwo H, Linehan M, Djelantik IG, Mercer D, et al. Climatic, temporal, and geographic characteristics of respiratory syncytial virus disease in a tropical island population. Epidemiol Infect 2008; 136:1319-27. doi: 10.1017/ S0950268807000015.

3. Khamis FA, Al-Kobaisi MF, Al-Areimi WS, Al-Kindi $\mathrm{H}$ Al-Zakwani I. Epidemiology of respiratory virus infections among infants and young children admitted to hospital in Oman. J Med Virol 2012; 84:1323-9. doi: 10.1002/jmv.23330.

4. Hall CB, Weinberg GA, Iwane MK, Blumkin AK, Edwards KM, Staat MA, et al. The burden of respiratory syncytial virus infection in young children. N Engl J Med 2009; 360:588-98. doi: 10.1056/NEJMoa0804877.

5. Dawson-Caswell M, Muncie HL Jr. Respiratory syncytial virus infection in children. Am Fam Physician 2011; 83:141-6.

6. Eisenhut M. Extrapulmonary manifestations of severe respiratory syncytial virus infection: A systematic review. Crit Care 2006; 10:R107. doi: 10.1186/cc4984.

7. Ng YT, Cox C, Atkins J, Butler IJ. Encephalopathy associated with respiratory syncytial virus bronchiolitis. J Child Neurol 2001; 16:105-8. doi: 10.1177/088307380101600207.

8. Playfor SD, Khader A. Arrhythmias associated with respiratory syncytial virus infection. Pediatr Anesth 2005; 15:1016-18. doi: 10.1111/j.1460-9592.2005.01580.x.

9. Hirayama K, Sakazaki H, Murakami S, Yonezawa S, Fujimoto K, Seto T, et al. Sequential MRI, SPECT and PET in respiratory syncytial virus encephalitis. Pediatr Radiol 1999; 29:282-6. doi: $10.1007 / \mathrm{s} 002470050589$.

10. Imamoğlu M, Cay A, Koşucu P, Ozdemir O, Cobanoğlu U, Orhan F, et al. Pneumatoceles in postpneumonic empyema: An algorithmic approach. J Pediatr Surg 2005; 40:1111-17. doi: 10.1016/j.jpedsurg.2005.03.048.

11. Fujii AM, Moulton S. Percutaneous catheter evacuation of a pneumatocele in an extremely premature infant with respiratory failure. J Perinatol 2003; 23:516-8. doi: 10.1038/sj.jp.7210964.

12. Fujii AM, Moulton S. VATS management of an enlarging multicystic pneumatocele. J Perinatol 2008; 28:445-7. doi: 10. 1038/sj.jp.7211928.

13. Randolph AG, Reder L, Englund JA. Risk of bacterial infection in previously healthy respiratory syncytial virus-infected young children admitted to the intensive care unit. Pediatr Infect Dis J 2004; 23:990-4. doi: 10.1097/01.inf.0000143647.88873.66.
14. Rihkanen H, Ronkko E, Nieminen T, Komsi KL, Räty R, Saxen H, et al. Respiratory viruses in laryngeal croup of young children. J Pediatr 2008; 152:661-5. doi: 10.1016/j.jpeds.2007.10.043.

15. Kirin BK, Topić RZ, Dodig S. Hepatitis during respiratory syncytial virus infection: A case report. Biochem Med (Zagreb) 2013; 23:112-6. doi: 10.11613/BM.2013.014.

16. Kim YN, You SJ. A case of acute necrotizing encephalopathy associated with parainfluenza virus infection. Korean J Pediatr 2012; 55:147-50. doi: 10.3345/kjp.2012.55.4.147.

17. Marco EJ, Anderson JE, Neilson DE, Strober JB. Acute necrotizing encephalopathy in 3 brothers. Pediatrics 2010; 125:e693-8. doi: 10.1542/peds.2009-1984.

18. Martin A, Reade EP. Acute necrotizing encephalopathy progressing to brain death in a pediatric patient with novel influenza A (H1N1) infection. Clin Infect Dis 2010; 50:e50-2. doi: $10.1086 / 651501$

19. Tabarki B, Thabet F, Al Shafi S, Al Adwani N, Chehab M, Al Shahwan S. Acute necrotizing encephalopathy associated with enterovirus infection. Brain Dev 2013; 35:454-7. doi: 10.1016/j.braindev.2012.07.001.

20. Ashtekar CS, Jaspan T, Thomas D, Weston V, Gayatri NA, Whitehouse WP. Acute bilateral thalamic necrosis in a child with mycoplasma pneumoniae. Dev Med Child Neurol 2003; 45:634-7. doi: 10.1111/j.1469-8749.2003.tb00969.x.

21. Park A, Suh SI, Son GR, Lee YH, Seo HS, Eun BL, et al. Respiratory syncytial virus-related encephalitis: Magnetic resonance imaging findings with diffusion-weighted study. Neuroradiology 2014; 56:163-8. doi: 10.1007/s00234-013-1305-z.

22. Tunkel AR, Glaser CA, Bloch KC, Sejvar JJ, Marra CM, Roos KL, et al. The management of encephalitis: Clinical practice guidelines by the Infectious Diseases Society of America. Clin Infect Dis 2008; 47:303-27. doi: 10.1086/589747.

23. Wu X, Wu W, Pan W, Wu L, Liu K, Zhang HL. Acute necrotizing encephalopathy: An underrecognized clinicoradiologic disorder. Mediators Inflamm 2015; 2015:792578. doi: 10.1155/ 2015/792578.

24. Davison C, Ventre KM, Luchetti M, Randolph AG. Efficacy of interventions for bronchiolitis in critically ill infants: A systematic review and meta-analysis. Pediatr Crit Care Med 2004; 5:482-9. doi: 10.1097/01.PCC.0000128891.54799.67. 